\title{
ON A SOLAR ORIGIN FOR THE COSMOGENIC NUCLIDE EVENT OF 775 A.D.
}

\author{
E. W. Cliver ${ }^{1}$, A. J. Tylka ${ }^{2}$, W. F. Dietrich ${ }^{3}$, AND A. G. Ling $^{4}$ \\ ${ }^{1}$ Space Vehicles Directorate, Air Force Research Laboratory, Sunspot, NM 88349, USA \\ ${ }^{2}$ Code 672, NASA Goddard Space Flight Center, Greenbelt, MD 20771, USA \\ ${ }^{3}$ Praxis, Inc., Alexandria, VA 22303, USA \\ ${ }^{4}$ Atmospheric Environmental Research, 3550 Aberdeen Ave., Kirtland AFB, NM 87117, USA \\ Received 2013 October 3; accepted 2013 November 16; published 2014 January 3
}

\begin{abstract}
We explore requirements for a solar particle event (SPE) and flare capable of producing the cosmogenic nuclide event of 775 A.D., and review solar circumstances at that time. A solar source for 775 would require a $>1 \mathrm{GV}$ spectrum $\sim 45$ times stronger than that of the intense high-energy SPE of 1956 February 23 . This implies a $>30 \mathrm{MeV}$ proton fluence $\left(F_{30}\right)$ of $\sim 8 \times 10^{10}$ proton $\mathrm{cm}^{-2}, \sim 10$ times larger than that of the strongest 3 month interval of SPE activity in the modern era. This inferred $F_{30}$ value for the $775 \mathrm{SPE}$ is inconsistent with the occurrence probability distribution for $>30 \mathrm{MeV}$ solar proton events. The best guess value for the soft X-ray classification (total energy) of an associated flare is $\sim \mathrm{X} 230\left(\sim 9 \times 10^{33} \mathrm{erg}\right)$. For comparison, the flares on 2003 November 4 and 1859 September 1 had observed/inferred values of $\sim \mathrm{X} 35\left(\sim 10^{33} \mathrm{erg}\right)$ and $\sim \mathrm{X} 45\left(\sim 2 \times 10^{33} \mathrm{erg}\right)$, respectively. The estimated size of the source active region for a $\sim 10^{34} \mathrm{erg}$ flare is $\sim 2.5$ times that of the largest region yet recorded. The 775 event occurred during a period of relatively low solar activity, with a peak smoothed amplitude about half that of the second half of the 20th century. The $\sim 1945-1995$ interval, the most active of the last $\sim 2000 \mathrm{yr}$, failed to witness a SPE comparable to that required for the proposed solar event in 775 . These considerations challenge a recent suggestion that the 775 event is likely of solar origin.
\end{abstract}

Key words: sun: particle emission - sun: flares

Online-only material: color figures

\section{INTRODUCTION}

Knowledge of the largest flares and solar proton events (SPEs) that the Sun can produce is important for both practical and theoretical reasons (Cliver \& Svalgaard 2004; Riley 2012; Schrijver et al. 2012; Aulanier et al. 2013). Here we consider a recent report of what is possibly the largest SPE during the last $\sim 10^{4} \mathrm{yr}$

From high-time-resolution (1-2 yr) measurements of ${ }^{14} \mathrm{C}$ in Japanese cedar trees from 750-820 A.D., Miyake et al. (2012) reported a $12 \%$ increase of carbon content from 774-775. Miyake et al. argued that both a nearby supernova and the Sun were improbable sources for the 775 event: a historical report (and/or remnant) would have been expected for a nearby supernova, while a solar origin would require a SPE much more intense than any observed so far. Subsequently, several sources, both solar and non-solar, have been suggested for the 775 event. The non-solar sources include a supernova hidden behind a dust cloud (Allen 2012) and galactic short-duration (Hambaryan \& Neuhäuser 2013) and long-duration gamma-ray bursts (Pavlov et al. 2013). Melott \& Thomas (2012) and Usoskin et al. (2013) favor a standard (eruptive flare; Reames 2013) solar source, and Eichler \& Mordecai (2012) proposed a non-traditional solar source-a superflare caused by a large comet colliding with the Sun. Recently, Miyake et al. (2013) have reported a second rapid ${ }^{14} \mathrm{C}$ increase from $992-993$ A.D. that was $\sim 0.6$ times as intense as the 775 event with a similar time profile and spectrum.

Usoskin \& Kovaltsov (2012) modeled the 775 event in terms of a $\sim 25-50$ multiple of the hard proton spectrum of the 23 February 1956 SPE. Usoskin et al. (2013) proposed a 45 times multiple and argued that "the Sun is to blame" for the 775 event. Given the current interest in determining the limits of extreme solar activity and the range of proposed origins for the 775 event, we examine the spectrum of the 1956 ground level event
(GLE; requiring $>500 \mathrm{MeV}$ protons) used to model the 775 cosmogenic nuclide event and review the solar circumstances that gave rise to the 1956 SPE. In addition we consider the production of large $>30 \mathrm{MeV}\left(F_{30}\right)$ events by the Sun during the modern era and the flare and active region sizes required to produce the 775 event. Our analysis is presented in Section 2 and results are summarized and discussed in Section 3.

\section{ANALYSIS}

\subsection{Spectrum of the 1956 February 23 GLE}

\subsubsection{High-energy Branch}

Seventy-one GLEs have been observed since 1956 (Cliver et al. 1982; Smart \& Shea 1991; Cliver 2006; Gopalswamy et al. 2013). The standard measure of GLE intensity is the percentage increase above background for a favorably located neutron monitor with a geomagnetic cut-off rigidity of $\sim 1 \mathrm{GV}$. Figure 1 is a histogram of GLE intensities. The 1956 February 23 GLE was the largest ever observed with a reported increase of $4554 \%$ versus $563 \%$ for the next biggest event.

Figure 2 shows energy spectra obtained by Tylka \& Dietrich (2009) for the nine GLEs from 1956 to the present with $>1 \mathrm{GV}$ proton (pr) fluence $\geqslant 10^{6} \mathrm{pr} \mathrm{cm}^{-2}$. The spectral form is a Band et al. (1993) function: a double-power-law form in energy that smoothly rolls one power-law into another. A proton energy of $\sim 430 \mathrm{MeV}(1 \mathrm{GV})$ is the approximate minimum energy required for a primary proton to produce ${ }^{10} \mathrm{Be}$ and ${ }^{14} \mathrm{C}$ at Earth (Beer et al. 2012). The $>1 \mathrm{GV}$ fluence of the 1956 event $\left(4.9 \times 10^{7} \mathrm{pr} \mathrm{cm}^{-2}\right.$; solid black line in Figure 2) is $\sim 6$ times that of the next largest event (1960 November $12 ; 8.0 \times 10^{6} \mathrm{pr} \mathrm{cm}^{-2}$ ) for which neutron monitor observations are available. The calculated ${ }^{14} \mathrm{C}$ production of $2.9 \times 10^{6}$ atoms $\mathrm{cm}^{-2}$ (Usoskin \& Kovaltsov 2012) for the 1956 GLE and the revised production of $1.3 \times$ $10^{8}$ atoms $\mathrm{cm}^{-2}$ obtained by Usoskin et al. (2013) for 775 A.D., 


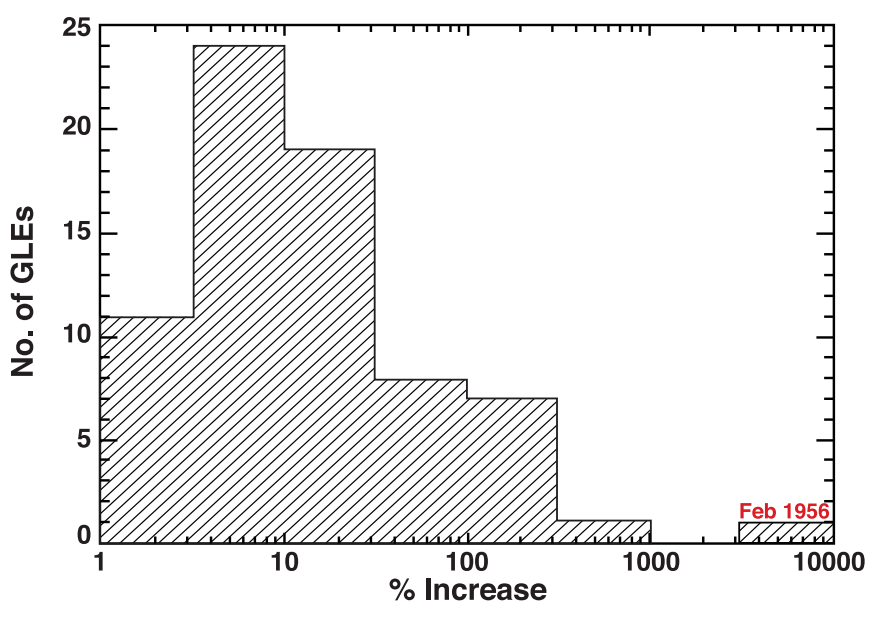

Figure 1. Histogram of the intensity of GLEs from 1942-2012.

implies that a $\sim 45$ times multiple of the 1956 SPE spectrum (dashed black line in Figure 2) is required for the 775 event.

\subsubsection{Low-energy Branch}

Because the 1956 SPE occurred before satellite observations of solar protons, the low-energy branch $(<430 \mathrm{MeV})$ of the spectrum is uncertain. As noted by Reedy (1977), the primary source of low-energy data points for events prior to 1963 is Webber et al. (1963). The list therein of SPE fluences during cycle 19 (1954-1964)—based on an amalgamation of groundbased ionospheric data and rocket and balloon observations-is largely used by all subsequent researchers. Of the biggest events there is a significant difference only for 1960 November 12 event for which Feynman et al. (1990) and Shea \& Smart (1990) list a $>30 \mathrm{MeV}$ fluence $\left(F_{30} ;>240 \mathrm{MV}\right)$ value of $9.0 \times 10^{9} \mathrm{pr} \mathrm{cm}^{-2}$ (see Masley \& Goedeke 1963), well above the $1.3 \times 10^{9} \mathrm{pr} \mathrm{cm}^{-2}$ value from Webber et al.

Usoskin et al. (2013) used their 45 times multiple of the 1956 February 23 spectrum and the Webber et al. (1963) value of $1.0 \times 10^{9} \mathrm{pr} \mathrm{cm}^{-2}$ at $>30 \mathrm{MeV}$ for this event to infer $F_{30}=$ $4.5 \times 10^{10} \mathrm{pr} \mathrm{cm}^{-2}$ for 775 .

Reedy (1977) analyzed the concentration of the cosmogenic nuclides ${ }^{22} \mathrm{Na}$ and ${ }^{55} \mathrm{Fe}$ (both with a half-lives of $\sim 2.5 \mathrm{yr}$ ) in lunar rocks and determined that the Webber et al. (1963) $F_{30}$ estimate of $9.4 \times 10^{9} \mathrm{pr} \mathrm{cm}^{-2}$ (adjusted upward from $8.9 \times$ $10^{10} \mathrm{pr} \mathrm{cm}^{-2}$ by inclusion of small events by others) for cycle 19 was too low by a factor of $\sim 5$. Subsequent additions of events (and a few changed $F_{30}$ values) common to the lists of Feynman et al. (1990) and Shea \& Smart (1990) increase the Webber et al. $F_{30}$ value to $1.0 \times 10^{10} \mathrm{pr} \mathrm{cm}^{-2}$. The most recent lunar-rockbased estimate of $F_{30}$ for cycle 19 is $2.3 \times 10^{10} \mathrm{pr} \mathrm{cm}^{-2}$ (Reedy 2012). Comparison of a list of riometer-based $>10 \mathrm{MeV} \mathrm{SPE}$ peak fluxes for cycle 19 from Bailey (1964) with those for common events in Webber et al. indicates a similar result; the median value of the ratios of the $>10 \mathrm{MeV}$ peak fluxes from Bailey to those of Webber et al. (1963) is 2.4 (cf. Reedy 1977).

Recently, Webber et al. (2007) revised the Webber et al. (1963) estimates of the three largest $F_{30}$ events of cycle 19, after considering a number of studies that had been published in the interim. The $F_{30}$ fluences for the three events were adjusted upward by factors of $\sim 1.3-3.0$ as follows: 1956 February $23,1.0 \Rightarrow 1.8 \times 10^{9} \mathrm{pr} \mathrm{cm}^{-2}, 1959$ July $10-17,3.2 \Rightarrow$ $4.0 \times 10^{9} \mathrm{pr} \mathrm{cm} \mathrm{cm}^{-2}$, and 1960 November $12-15,2.0 \Rightarrow$ $6.0 \times 10^{9} \mathrm{pr} \mathrm{cm}^{-2}$.

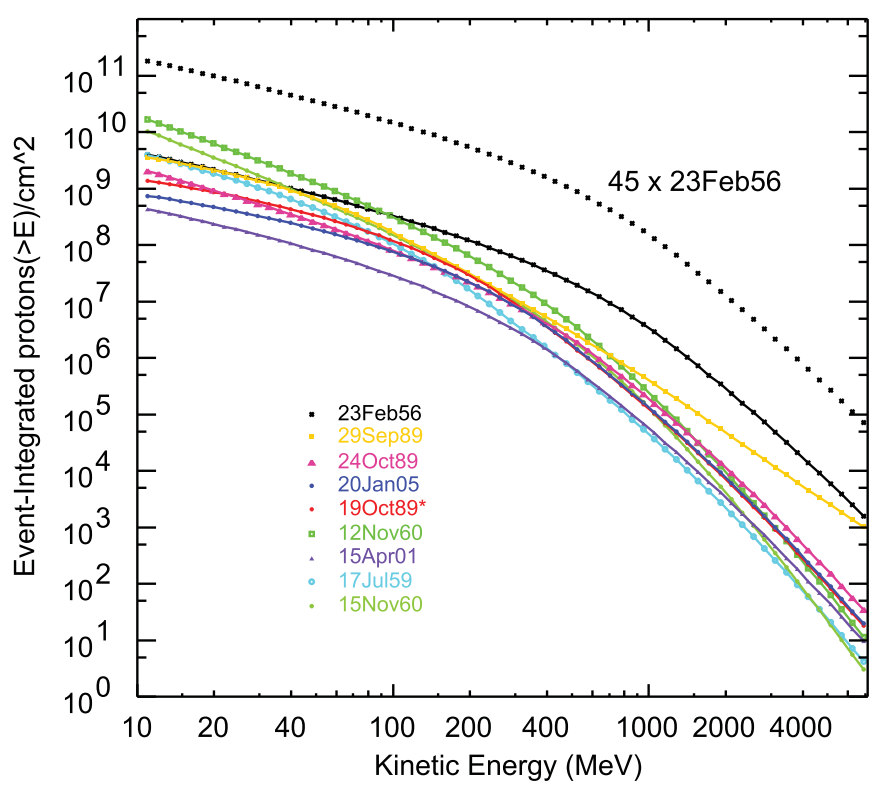

Figure 2. Spectra of the nine GLEs from 1956-2012 with $>1$ GV fluences $\geqslant 10^{6} \mathrm{pr} \mathrm{cm}^{-2}$. For the events of 1956-1960, the Band fits reflect the increased $>10,>30$, and $>100 \mathrm{MeV}$ fluence estimates from Webber et al. (2007). The Band fit for 1989 October 19 includes only the first $19 \mathrm{hr}$ of the event.

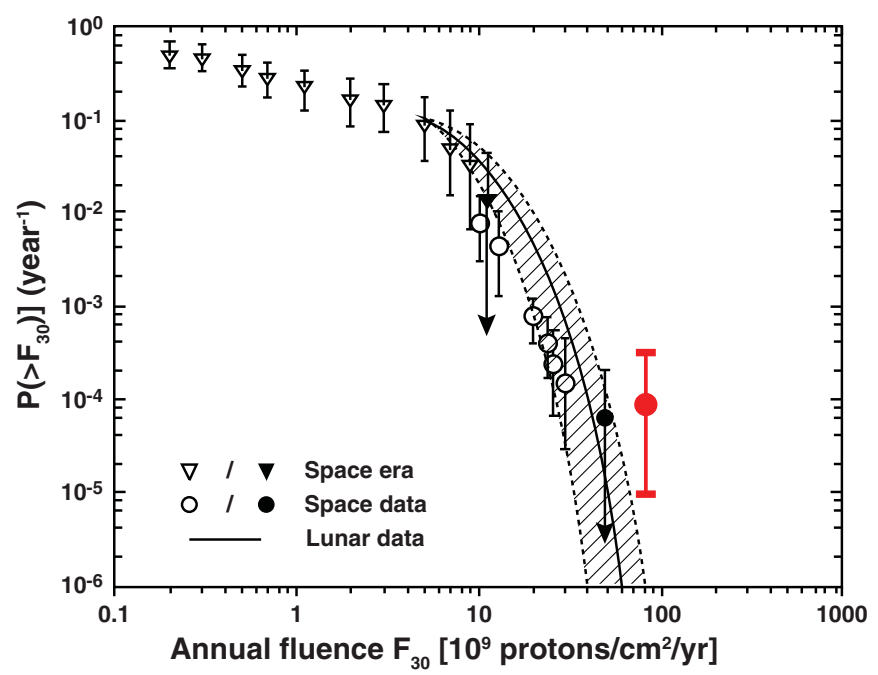

Figure 3. OPDF for $>30 \mathrm{MeV}$ SEP events with the red point for the revised estimate of the 775 event added (adapted from Kovaltsov \& Usoskin 2014, with permission of Solar Physics).

Of primary interest here is the revised $F_{30}$ value for 1956 February 23. It increases the $F_{30}$ estimate for the 775 event from $\sim 4.5 \times 10^{10}$ to $\sim 8 \times 10^{10} \mathrm{pr} \mathrm{cm}^{-2}$. We regard this as a conservative estimate because the 1.8 multiplier used by Webber et al. falls below both the $\sim 2.0$ ratio of the Bailey (1964) to Webber et al. (1963) peak $>10 \mathrm{MeV}$ fluxes for this event and the 2.3 ratio of the solar cycle $19 F_{30}$ value from Reedy (2012) to that of Webber et al. (1963). As can be seen in Figure 3, the red point corresponding to the revised $F_{30}$ value of $\sim 8 \times 10^{10} \mathrm{pr} \mathrm{cm}^{-2}$ for 775 falls outside the $90 \%$ confidence interval (Gehrels 1986) of the occurrence probability distribution function (OPDF) recently constructed by Kovaltsov \& Usoskin (2014) for $>30 \mathrm{MeV}$ SPEs. This OPDF is based on direct satellite measurements of SPEs during the space age, measurements of sharp increases of ${ }^{14} \mathrm{C}$ and ${ }^{10} \mathrm{Be}$ concentration in tree rings and ice cores during the last 11,400 yr, and 
Table 1

Estimates of $F_{30}$ for Large Composite Events During Cycle 19

\begin{tabular}{lccccc}
\hline \hline Date & Webber et al. (1963) & Riometer (Scaled) & Lunar Rock (Scaled) & Webber et al. (2007) & Average Value \\
\hline $1959 \mathrm{Jul}$ & $3.2^{\mathrm{a}}$ & 9.0 & 7.4 & 4.0 & $\sim 7$ \\
$1960 \mathrm{Nov}$ & 2.1 & 4.3 & 4.8 & 6.0 & $\sim 5$
\end{tabular}

Note. ${ }^{a}$ Units for all values are $10^{9} \mathrm{pr} \mathrm{cm}^{-2}$.

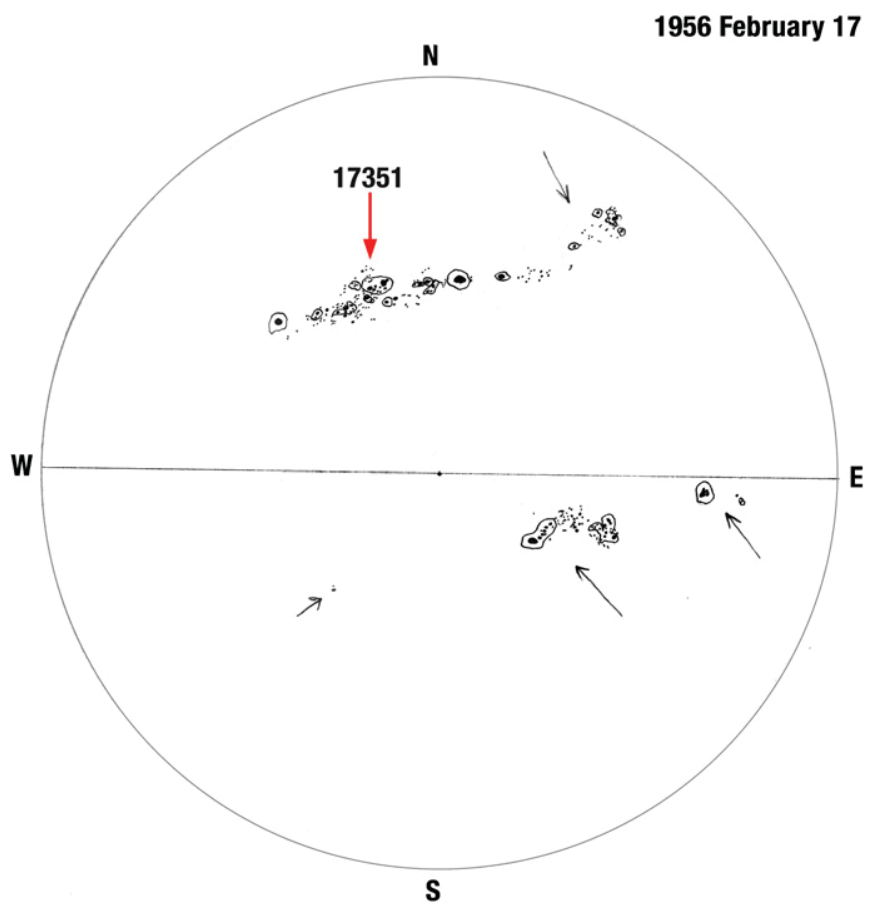

Figure 4. Sunspot drawing for 1956 February 17 showing the source region (Greenwich 17351; red arrow) of the 1956 February GLE.

(A color version of this figure is available in the online journal.)

cosmogenic nuclides in lunar rocks during the last half-million years.

\subsection{Solar Circumstances for the 1956 \\ February 23 GLE-associated Flare}

Figure 4 shows the sunspot drawing from Sacramento Peak Observatory for 1956 February 17, when Greenwich active region 17351 was near disk center (N22W02) with a corrected (for foreshortening) spot area of 1393 millionths of a solar hemisphere (msh). It was part of a noteworthy string of six active regions spanning $\sim 60^{\circ}$ of heliolatitude at $\sim$ N20 with a combined area of $3809 \mathrm{msh}$. The GLE-associated flare from region 17351 on February 23 (N23W80) had its $\mathrm{H} \alpha$ maximum (class 3) at 03:42 UT. The sunspot number (SSN) for February 23 was 177 . The 1956 SPE occurred during solar cycle 19, the largest cycle (peak 13 month smoothed SSN of 201.3) since sunspot recording began in 1610. Cycle 19 occurred during the strongest $\sim 50$ yr interval of solar activity $(\sim 1945-1995)$ in the last $\sim 2000 \mathrm{yr}$ (Usoskin et al. 2007).

In contrast to the $1956 \mathrm{SPE}$, the cosmogenic nuclide event of 775 occurred during a period of inferred low solar activity. Figure 5, adapted from Usoskin et al. (2007), contains a smoothed decadal record of solar activity based on ${ }^{14} \mathrm{C}$ concentration in tree rings for the last $6000 \mathrm{yr}$. The interval, indicated by a black oval, in which the 775 event occurred has a peak ${ }^{14} \mathrm{C}$-based-SSN of only about 25 compared to $\sim 70$ for the epoch in which the 1956 SPE occurred (red oval). While recent re-analysis of the SSN (Cliver et al. 2013) indicates that the reconstructed SSN in this figure is understated by $\sim 50 \%$ before $\sim 1885$, the relatively quiet characterization of the period ca. 775 still applies. It has been suggested (McCracken et al. 2004) that large SPEs might occur preferentially during periods of low solar activity but the nitrate-in-ice-cores proxy for SPEs on which this picture was based has recently been invalidated by Wolff et al. (2012).

Usoskin et al. (2013) noted that the 775 event was accompanied by a cluster of low-latitude aurora reported from Shanxi Province, China, in 770 (twice), 773, and 775 based on the compilations of Keimatsu (1973) and Yau et al. (1995). Of these four events, Yau et al. (1995), who expunged many unreliable records of aurora given in the Keimatsu catalog, only list the two 770 events. From a recent re-examination of Keimatsu (1973), F. R. Stephenson (2013, private communication) notes that for the two 770 events, there is no mention that the phenomenon occurred at night, making it doubtful whether aurora are being referred to. He suggests, however, that a report of "more than 10 bands of white vapor" on January 12776 may be auroral in nature. In either case, the evidence for a grouping of strong auroral activity ca. 775 is weakened.

\subsection{Historical Cases of Large $>30 \mathrm{MeV}$ Fluence SPEs}

During the satellite era, the largest $F_{30}$ events have been produced by sequences of eruptive flares originating near solar central meridian (Smart et al. 2006; Cliver \& Dietrich 2013). Prominent examples of such sequences occurred in 1972 August $\left(5.0 \times 10^{9} \mathrm{pr} \mathrm{cm}{ }^{-2}\right.$; Shea \& Smart 1990) and 1989 October $\left(4.3 \times 10^{9}\right)$. Both of these episodes exhibited a strong shock spike in their $F_{30}$ time-intensity profile. Table 1 gives various $F_{30}$ values for similar composite events in cycle 19: 1959 July and 1960 November. In addition to the initial Webber et al. (1963) $F_{30}$ value, there are values that were: (1) obtained by scaling up the Webber et al. values by the ratios of the peak $>10 \mathrm{MeV}$ proton fluxes reported by Bailey (1964) and Webber et al. (1963), (2) scaled up from the Webber et al. (1963) values by the factor of 2.3 based on the analysis of cosmogenic nuclides in lunar rocks (Reedy 2012), and (3) determined by Webber et al. (2007). From (1)-(3) we take average values of $\sim 7 \times 10^{9} \mathrm{pr} \mathrm{cm}^{-2}$ and $\sim 5 \times 10^{9} \mathrm{pr} \mathrm{cm}^{-2}$ for 1959 July and 1960 November, respectively. Since the time resolution of tree ring records is $1 \mathrm{yr}$, we considered the strong activity from 1989 August-October, including five GLEs, as a single event to obtain a worst case scenario in modern times. The $F_{30}$ for the 3 month interval from 1989 August 12 to November 10 was $7.2 \times 10^{9} \mathrm{pr} \mathrm{cm}^{-2}$. Thus a $F_{30}=\sim 8 \times 10^{10} \mathrm{pr} \mathrm{cm}^{-2}$ event for 775 A.D., based on a 45 times multiple of the updated (Webber et al. 2007) $F_{30}$ value for the 1956 event, would be $\sim 10$ times larger than any event of the modern era. 


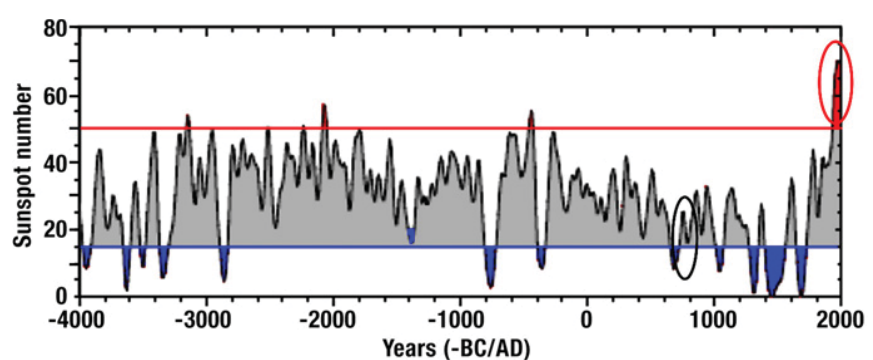

Figure 5. ${ }^{14} \mathrm{C}$-based decadal-smoothed SSN for the last $6000 \mathrm{yr}$ (adapted from Usoskin et al. 2007, with permission of Astronomy \& Astrophysics). Intervals of low solar activity ca. 775 and high activity ca. 1956 are indicated by black and red ovals, respectively. The solid blue (red) line delineates grand minima (maxima)

(A color version of this figure is available in the online journal.)

\subsection{Energy Requirements for a Solar Flare and Active Region in 775 A.D.}

Cliver \& Dietrich (2013) obtained relationships, with broad scatter, between (1) $F_{30}$ and 1-8 $\AA$ soft X-ray (SXR) fluence and (2) flare bolometric energy and SXR fluence, that give a best guess estimate of total energy of $\sim 9 \times 10^{33}$ erg for a 775 flare versus $\sim 2 \times 10^{33} \mathrm{erg}$ for the Carrington event. This estimate is based on the $F_{30}$ value of $\sim 8 \times 10^{10} \mathrm{pr} \mathrm{cm}^{-2}$ for the 775 event and the flare energy-partition work of Emslie et al. (2012) who found that, on average, mass ejection kinetic energy $=\sim 3$ times flare bolometric energy. From Figure 5 in Veronig et al. (2002) which relates SXR fluence to intensity, we infer a flare classification of $\sim$ X230 (versus $\sim X 45$ for the 1859 event). Corresponding values for the 2003 November 4 flare are $\sim 10^{33}$ erg and $\sim$ X35 (Emslie et al. 2012; Cliver \& Dietrich 2013). Emslie et al. (2012) estimated a free magnetic energy of $2.9 \times 10^{33} \mathrm{erg}$ for NOAA region $486(2610 \mathrm{msh}$ on 2003 October 29). Scaling linearly by sunspot area (assuming an energy conversion efficiency of $\sim 0.5$; Emslie et al. 2012) implies an active region for the 775 event with area of $\sim 16,000 \mathrm{msh}$, $\sim 2.5$ times the size of the largest region yet recorded $(6132 \mathrm{msh}$ on 1947 April 8).

\section{SUMMARY AND DISCUSSION}

The inferred specifications for a 775 SPE, associated eruptive flare, and source active region are: (1) a multiple of $\sim 45$ times the intense hard spectrum of the 1956 February 23 SPE (Usoskin et al. 2013); (2) a $>30 \mathrm{MeV}$ proton fluence of $\sim 8 \times 10^{10} \mathrm{pr} \mathrm{cm}^{-2}$ $(\sim 10$ times larger than that for the strongest 3 month interval of activity during the modern era (1989 August-October)); (3) a best guess SXR class of $\sim$ X230 with total energy $\sim 9 \times 10^{33} \mathrm{erg}$ ( $\sim 4-5$ times the energy estimate for the Carrington event and 9 times 2003 November 4); and (4) an active region with sunspot area of $\sim 16,000 \mathrm{msh}, \sim 2.5$ times that of 1947 April). The ${ }^{14}$ C-based smoothed SSN ca. 775 had a peak amplitude about half that of the 1945-1995 interval.

The high multiple of the 1956 February spectrum required to produce the $775{ }^{14} \mathrm{C}$ event presents a challenge for the attribution of 775 to a solar source. The 1956 February GLE is the strongest yet recorded. It occurred during the most active period of the last $\sim 2000 \mathrm{yr}$ while 775 fell during a period of relatively low solar activity. Given the revised $F_{30}$ value for 1956 (Webber et al. 2007), the point for 775 falls outside the $>30 \mathrm{MeV}$ OPDF of Kovaltsov \& Usoskin (2014). The $775 F_{30}$ value might be reduced by a streaming limit (Reames 2013), but such a reduction can be offset/negated by contributions from delayed shock spikes and/or closely spaced SPEs (Cliver
$\&$ Dietrich 2013). The recent discovery of a second large ${ }^{14} \mathrm{C}$ event in 993 makes a solar source for 775 more attractive-and prospects for a more exotic source such as a short-duration gamma-ray burst (Hambaryan \& Neuhäuser 2013) less likely (Miyake et al. 2013). That said, solar conditions in 993 A.D., on the cusp of a grand minimum (Figure 5), were less promising than those of 775. On balance, it seems pre-mature to rule out a non-solar source for 775. As pointed out by Miyake et al. (2013), analysis of more high-time-resolution cosmogenic nuclide data is needed.

E.W.C. thanks Karel Schrijver and Jürg Beer for organizing three ISSI workshops on Extreme Solar Events and acknowledges support from AFOSR. We thank Ilya Usoskin for helpful comments.

\section{REFERENCES}

Allen, J. 2012, Natur, 486, 473

Aulanier, G., Démoulin, P., Schrijver, C. J., et al. 2013, A\&A, 549, A66 Bailey, D. K. 1964, P\&SS, 12, 495

Band, D., Matteson, J., Ford, L., et al. 1993, ApJ, 413, 281

Beer, J., McCracken, K., \& von Steiger, R. 2012, Cosmogenic Radionuclides: Theory and in the Terrestrial and Space Environments (Berlin: Springer) Cliver, E. W. 2006, ApJ, 639, 1206

Cliver, E. W., Clette, F., \& Svalgaard, L. 2013, CEAB, 37, 401

Cliver, E. W., \& Dietrich, W. F. 2013, JSWSC, 3, A31

Cliver, E. W., Kahler, S. W., Shea, M. A., \& Smart, D. F. 1982, ApJ, 260, 362

Cliver, E. W., \& Svalgaard, L. 2004, SoPh, 224, 407

Eichler, D., \& Mordecai, D. 2012, ApJL, 761, L27

Emslie, G., Dennis, B. R., Shih, A. Y., et al. 2012, ApJ, 759, 71

Feynman, J., Armstrong, T. P., Dao-Gibner, L., \& Silverman, S. 1990, JSpRo, 27,403

Gehrels, N. 1986, ApJ, 303, 336

Gopalswamy, N., Xie, H., Akiyama, S., et al. 2013, ApJL, 765, L30

Hambaryan, V. V., \& Neuhäuser, R. 2013, MNRAS, 430, 32

Keimatsu, M. 1973, A Chronology of Aurorae and Sunspots Observed in China, Korea and Japan, 6-8 Century, Part IV, Vol. 10 (Kanazawa: Ann. Sci. College of Liberal Arts, Kanazawa Univ.)

Kovaltsov, G. A., \& Usoskin, I. G. 2014, SoPh, 289, 211

Masley, A. J., \& Goedeke, A. D. 1963, in Proc 3rd Int. Space Science Symp., Life Sciences and Space Research III, ed. W. Priester (Amsterdam: North Holland), 95

McCracken, K. G., Dreschhoff, G. A. M., Smart, D. F., \& Shea, M. A. 2004, SoPh, 224, 359

Melott, A. L., \& Thomas, B. C. 2012, Natur, 491, E1

Miyake, F., Masuda, K., \& Nakamura, T. 2013, NatCo, 4, 1748

Miyake, F., Nagaya, K., Masuda, K., \& Nakamura, T. 2012, Natur, 486, 240

Pavlov, A. K., Vdovina, M. A., Vasilyev, G. I., et al. 2013, MNRAS, 435, 2878 Reames, D. V. 2013, SSRv, 175, 53

Reedy, R. C. 1977, in Proc. 8th Lunar Sci. Conf., Vol. 1, The Moon and the Inner Solar System, ed. R. B. Merrill (New York: Pergamon), 825

Reedy, R. C. 2012, in Proc. 43rd Lunar \& Planet. Sci. Conf., Update on SolarProton Fluxes During the Last Five Solar Activity Cycles (Houston, TX: Lunary \& Planetary Institute), 1285

Riley, P. 2012, SpWea, 10, S02012

Schrijver, C. J., Beer, J., Baltensperger, U., et al. 2012, JGR, 117, A08103

Shea, M. A., \& Smart, D. F. 1990, SoPh, 127, 297

Smart, D. F., Shea, M. A., Spence, H. E., \& Kepko, L. 2006, AdSpR, 37, 1734 Tylka, A., \& Dietrich, W. 2009, in Proc. 31st Int. Cosmic Ray Conf., ed. M. Giller \& J. Szabelski (£ódź, Poland: Universal Academy Press), ICRC0273

Usoskin, I. G., \& Kovaltsov, G. A. 2012, ApJ, 757, 92

Usoskin, I. G., Kromer, B., Ludlow, F., et al. 2013, A\&A, 552, L3

Usoskin, I. G., Solanki, S. K., \& Kovaltsov, G. A. 2007, A\&A, 471, 301

Veronig, A., Temmer, M., Hanslmeier, A., Otruba, W., \& Messerotti, M. 2012, A\&A, 382, 1070

Webber, W. R., Benbrook, J. R., Thomas, J. R., Hunting, A., \& Duncan, R. 1963, Boeing Report (Seattle, Washington: The Boeing Company of Seattle), D290469

Webber, W. R., Higbie, P. R., \& McCracken, K. G. 2007, JGR, 112, A10106

Wolff, E. W., Bigler, M., Curran, M. A. J., et al. 2012, GeoRL, 39, L08503

Yau, K., Stephenson, F., \& Willis, D. 1995, A Catalogue of Auroral Observations from China, Korea and Japan (193 B.C.-A.D. 1770), Technical Report RALTR-95-073 (Chilton, Oxfordshire: Rutherford Appleton Laboratory) 